\title{
Review on the Effect of Resveratrol on Central Nervous System Disease ${ }^{\#}$
}

\author{
Wenzhe Dong1, Yan Zhou1, Zhifang Yang2* \\ ${ }^{1}$ Department of Pharmacology, Shanghai University of Medicine \& Health Sciences, Shanghai, China \\ ${ }^{2}$ School of Basic Medical Sciences, Shanghai University of Medicine \& Health Sciences, Shanghai, China \\ Email: *guicaitang@163.com
}

Received 1 February 2016; accepted 22 March 2016; published 25 March 2016

Copyright (C) 2016 by authors and Scientific Research Publishing Inc.

This work is licensed under the Creative Commons Attribution International License (CC BY).

http://creativecommons.org/licenses/by/4.0/

(c) (i) Open Access

\begin{abstract}
Resveratrol (3,5,4'-trihydroxy-trans-stilbene, RV) is a kind of phytoalexin found in many kinds of plants and food. As a natural antioxidant, RV shows significant biological activity, including anti-tumor activity, antitubulin activity, cardiovascular disease resistance activity, etc. Many experiments confirm that resveratrol displays a wide range of beneficial effects on human diseases including heart disease and cancer, especially in the treatment of central nervous system disease, such as Huntington's disease (HD), Alzheimer's disease (AD) and Parkinson's disease (PD). This paper summarizes the positive effects of $\mathrm{RV}$ on several central nervous system diseases.
\end{abstract}

\section{Keywords}

\section{Resveratrol, Central Nervous System Diseases, Disease Treatment, Alzheimer's Disease}

\section{Introduction}

Resveratrol (3,5,4'-trihydroxystilbene, $\left.\mathrm{C}_{14} \mathrm{H}_{12} \mathrm{O}_{3}\right)$ is a type of natural phenol, and a natural phytoalexin produced by several plants in response to injury or pathogens. It exists as two geometric isomers: cis-( $\mathrm{Z}$ ) and trans-(E) (Figure 1). Many everyday food ingredients contain resveratrol, such as peanut, the grape (red), giant knotweed, mulberry and other plants [1], and it is also found in red wine since the highest content of resveratrol is in grapes and grape skins, which increases the likelihood of its effect on the wider population. While RV content in plants is relatively low, and extract cost is high, chemical synthesis of resveratrol has become a major means of its development.

Resveratrol (RV) is a kind of natural antioxidant. Since the first article about the potential positive effect of $\mathrm{RV}$ on cancer in mice was published [1], the simple micromolecule attracted a lot of attention. Resveratrol

${ }^{\#}$ Supported by Shanghai Science and Technology Development Funds (Fund No. B230214001).

${ }^{*}$ Corresponding author. 


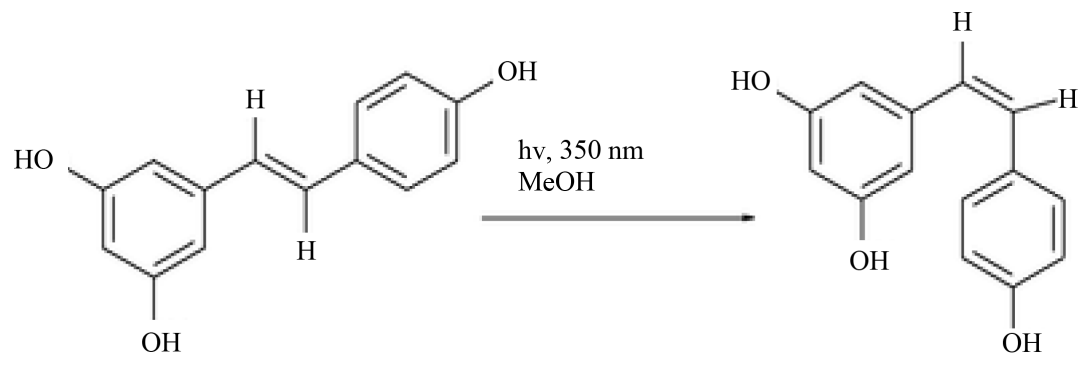

Figure 1. Chemical structure of Resveratrol ((3,5,4'-trihydroxystilbene, $\left.\mathrm{C}_{14} \mathrm{H}_{12} \mathrm{O}_{3}\right)$, transform (Left) can undergo isomerization to the cis-form (Right) when exposed to ultraviolet irradiation.

shows significant biological activity, including anti-tumor activity, antitubulin activity, cardiovascular disease resistance activity, etc. Therefore, more people focus on resveratrol, and scientific research groups and companies invest huge energy in the development of related food additives, cosmetics and the so-called natural medicine.

There have been a number of clinical studies on human, and in the next two to five years more related researches are expected to be conducted. At that time, we will know more about resveratrol's biological effect on human. In a large number of in vitro and in vivo experiments, RV has many beneficial effects on human disease, but the mechanisms are unclear. After oral administration, RV enters the circulatory system and is distributed in various tissues and organs. At the same time RV can quickly go through the blood brain barrier and be distributed in the brain tissue [2]. But the drug has its limitation of use. It can be metabolized quickly in the body and become a product with unknown activity, so the oral bioavailability is very low [2].

RV has definite effect on many molecules, but the mechanism of the influence on human health seems to be more related with its antioxidant activity. It is known that the oxidative stress is associated with a variety of central nervous system lesions, so a lot of researches in recent years have focused on RV application in treatment of a variety of central nervous system lesions, such as Alzheimer's disease (AD), Huntington's disease (HD), Parkinson's disease (PD), etc. [3].

This article intends to review the positive effect of RV on several kinds of central nervous system lesions, including Alzheimer's disease, Huntington's disease, Parkinson's disease, etc., and summarize the functional mechanism of RV on these diseases in the existing studies.

\section{RV and Alzheimer's Disease}

Alzheimer's disease is a progressive, age-dependent and neurodegenerative disorder, and the most common cause of Senile Dementia. The pathological and histological study of the brains of patients suffering from AD shows that the cortex and hippocampus area has ultrastructure changes, and two classic injuries are triggered, including senile plaques mainly composed of $\mathrm{A} \beta$ peptide and intracellular neurofibrillary tangles composed of excessively phosphorylated tau protein. Tau protein is a kind of multi-functional microtubule associated protein, which mainly acts on the collection of microtubules and form these polymers by bridging with other fibrous skeleton [4] [5]. In the brains of patients suffering from $\mathrm{AD}$, the earliest found variation is structure and conformation changes of Tau protein caused by the excessive phosphorylation of Tau protein under the action of different protein kinase and phosphatase systems [6], which influence the combination with tubulin and promote the capacity set of microtubules [7]. The most relevant protein kinase which participates in the neuronal degenerationinduced by Tau protein is glycogen synthase kinase 3 (GSK 3) [8]. GSK 3 increases the excessive phosphorylation of Tau protein loci, so that Tau protein transforms into a kind of protein not associated with the cytoskeleton.

Although most of the cases of Ad are incidental and the etiology is unknown, some familial genetic genes are closely related to the disease, such as amyloid precursor peptide (APP), presenilin-1 (PS1) and presenilin-2 (PS2). In the two kinds of cases (familial and occasional), the pathological changes associated with APP are considered to be related to $\mathrm{A} \beta$ amyloid peptide protein. Due to the formation of diffusible $\beta$ amyloid protein oligomer, fiber and amyloid protein plaques can be gathered and formed, and synaptic dysfunction and $\mathrm{AD}$ toxic 
effect can be reduced [9]. In neuropathology study, the cadaver specimen test of patients with AD shows that their neuron apoptosis rate increases [10]. Cell apoptosis is the behavior of active cell killing in a certain order related to the activity of caspase. In the nervous system, apoptosis seems to be caused by the lack of nutrition factors. The lack of nutritional factors for intracellular signaling pathway transduction triggers the activity of caspase. Due to the lack nutrition factors in the nervous system, BAD and Bcl-2 Bcl-2 will interact with each other and be phosphatased, so that the release of mitochondrial cytochrome $\mathrm{C}$ and apoptosis factors increase, and eventually caspase 3 is activated [11]. These activities in the motor neurons will trigger oxidative stress, and produce a series of products such as nitric oxide, oxide and nitrite. These products can also activate caspase 3, and contribute to a wider range of cell apoptosis. In the earlier study, it is recognized that presenilin also adjusts neuronal apoptosis. RV can also activate SIRT1 [12], reduce $\mathrm{A} \beta$ product by relying on silent regulatory proteins, and eventually inhibit the formation of $\mathrm{A} \beta$ amyloid protein fibers [13]. In addition, results of in vitro test show that SIRT1 can directly make Tau protein deacetylated. The removal of these acetyls may expose ubiquitinligating enzyme with lysine residues, so that Tau protein may be marked as proteasome and degraded [14].

The research of Hooper et al. has shown that P53 of superior temporal gyrus with AD will be tripled, and P53 can indirectly induce the Tau protein phosphorylation. Therefore, P53 has good therapeutic effect on AD. This effect may be related to the adjustment of cell death pathways (in other related nervous system lesions it has the same effect). P53 is the substrate of SIRT1. This discovery further confirms that RV can delay the progress of $\mathrm{AD}$.

\section{RV and Huntington's Disease}

$\mathrm{HD}$ is an autosomal dominant inheritance disease of the nervous system; and the most significant pathological manifestation of the disease is the progressive decrease of striatal neurons, causing abnormal movements and cognitive decline [15]. The origin of Huntington's disease is the repeated duplication of HTT on N terminal in an unstable trinucleotide CAG gene [16]. The JTT product is the variant HTT polyglutamine. Abnormal protein and protein act upon each other. Due to such, cell toxic aggregates are formed on neurons [17]. The function defect of neurons in HTTP fragments is the pathological manifestation of HD.

3 -nitropropionic acid is a kind of inhibitor of electron transport chain. This kind of electron transport chain induces phenotypic symptoms of HD. Resveratrol effectively inhibits the effect of 3-nitropropionic acid. This inhibiting effect can help patients to reduce the production of HD neurotoxin. RV inhibits the activity of cyclooxygenase I (COX), thereby significantly improving the motor and cognitive disorder of HD induced by 3-nitropropionic acid [18]. In addition, through activation of SIRT1, RV prevents the mutational polyglutamine from producing neurotoxin and injuring neurons [19]. A variety of mechanisms have been put forward in the previous studies. M-HTT may trigger the striatum nerve degeneration, including mitochondrial dysfunction, oxidative stress and cell apoptosis. In this case, the activation of P53 plays a crucial role in preventing the toxicity of M-HTT in human nerve cells. In the experiment of transgenic mice, no matter whether the inhibition effect of tumor suppressor gene P53 has phenotype, its HD cell dysfunction can be mediated and its function of cell division can be impaired [20]. The deacetylation effect of P53 can reduce its activity and inhibit the dependent apoptosis of P53. RV can affect P53's deacetylation effect by activating SIRT1. So RV reduces M-HTT's toxicity for cells, thus protecting cells. The protection effect is realized by enhancing SIRT1 activity or indirectly inducing P53's inhibition of toxic effect [21]. Generally, the effect of P53 on HD is associated with the enhancement of mitochondrial oxidation [22], and activation of SIRT1 occurs when RV exists and cell apoptosis is allowed to adapt to energy shortage [23].

$\mathrm{RV}$ can be effectively inserted into the mitochondrial oxidation through its resistance to oxidation, and offset impaired mitochondrial function by activating SIRT1-PGC1 $\alpha$ passage [24]. In fact, PGC1 $\alpha$ adjusts the expression and activity of ROS, thus removing antioxidant enzyme activity and offsetting oxidative stress [25].

\section{RV and Parkinson's Disease}

In addition to Alzheimer's disease, PD is the most common neurodegenerative disease. The prevalence rate of people over the age of 65 in the developed countries is nearly $2 \%$ [26]. Although we know little about the etiology of the occasional PD, some evidences still show that environmental factors and genetic tendency promote its development. Take $\alpha$ synaptic nucleoprotein gene as an example. The large genome missense mutation or frequent proliferation will cause autosomal dominant Parkinson's syndrome. Clinically, PD is characterized by 
progressive neurodegenerative disorder, including muscle rigidity, tremor and bradykinesia. And in extreme case action ability is completely lost. The root cause of motor symptoms is the degeneration of dopaminergic neurons in the substantianigra and the accumulation of intracytoplasmiclewy body (including $\alpha$ synuclein and ubiquitin) [27]. After dopamine is inactivated byoxidase (MAO), excessive hydrogen peroxide is produced. The hydrogen peroxide must be continuously detoxified by intracellular antioxidant.

It is generally believed that the manner of death of dopamine cells is cell apoptosis, rather than necrosis, but even this basic concept is still controversial; there is no doubt that oxidation and nitration stress of substantianigra is the outstanding characteristic of the disease.

Nitride (nitric oxide and peroxynitrite) is closely related to the change of the activity of iNOS. By contrast, the source of oxygen radical is not so clear, mainly based on indirect biochemical changes, such as higher level of iron, the change of antioxidant mechanism and mitochondrial dysfunction.

Mitochondrial injury is involved in the pathogenesis of PD. The related theory was established more than 20 years ago. Compound I inhibitor is known as the main source of free radicals, and it is thought that change to its function may be beyond the reduction of ATP and cause the increase of oxidative stress, thus explaining the occurrence of the disease.

RV can improve mitochondrial respiratory function through SIRT1-AMPK and PGC-1 $\alpha$ pathway. It plays an important role in the treatment. To be specific, RV activates AMPK-SIRT1signal pathway, so as to induce the activity of PGC-1 $\alpha$ [28]. PGC-1 $\alpha$ 's influence on mitochondrial respiratory capacity leads to the increase of mitochondrial biosynthesis and improvement of mitochondrial function.

An interesting assumption for PD neuron group is about ATP attenuation and internal flow of calcium ions. Monoamine oxidase induces the conduction of calcium produced by dopamine metabolism through reactive oxygen (mainly hydrogen peroxide) in astrocytes [29]. This creates the metabolic stress, because calcium entering the cells repeatedly and persistently needs to be balanced by ATP pump, so as to restore calcium homeostasis. It has been confirmed that the opening of L-form calcium channel of this type of neurons of mitochondria makes them very vulnerable to infection [30]. RV can effectively prevent the increase of calcium and affect PD process [31].

In the experimental model of PD, RV's protection of dopaminergic neurons may be associated with the resistance to oxidation of drugs. RV's removal of hydrogen peroxide $\left(\mathrm{H}_{2} \mathrm{O}_{2}\right)$ may be particularly effective; when the drug dose is $100 \mu \mathrm{g} / \mathrm{mL}, 60 \%$ of its effect is shown. In addition, RV induces SIRT1 activity expression, which can prevent the accumulation of pathological $\alpha$ synuclein [32]; to be specific, SIRT1 can make heat shock factor 1 (HSF1) deacetylated and activated, thus affecting molecules and molecular chaperone, including the transcription of heat shock protein 70 (HSP70). HSP70 regulates cell protein balance and reduces the abnormal protein aggregation.

Research has shown that GSK-3 $\beta$ inhibitor can prevent dopaminergic neurons from injury induced by all kinds of emergency. This is because $\alpha$ synuclein is the substrate of GSK-3 $\beta$ phosphorylation. Therefore, GSK-3 $\beta$ is associated with the pathogenesis of PD [33]. In the PD cell model, RV can reduce the expression of $\alpha$ synuclein by partially inhibiting GSK-3 $\beta[34]$.

\section{Conclusions}

In recent studies, it is uncovered that mitochondrial metabolism and reactive oxygen imbalance are the major causes of many neurodegenerative diseases. However, whether these two factors are the main influence factors of disease is still unknown. Some small molecules show the ability to adjust reactive oxygen, metal poisoning and abnormal protein aggregation. Evaluation of the ability and safety of these small molecules is the important factor and strategy of the development of new treatment for neurodegenerative diseases.

As the compound with various neuroprotective effects, RV has potential application in the treatment of nerve injury. It is particularly attractive that recent studies show that through SIRT1/AMPK/PGC1 $\alpha$ channel, RV can improve mitochondrial function and biosynthesis. Therefore the benefit of RV is not only limited to the antioxidant and anti-inflammatory properties.

But there are problems if we use RV as a possible drug for the treatment purpose. The water solubility of RV is low. Although clinical trials have shown that it is well tolerated in human during the trials but long-term effects of using resveratrol are currently unknown. Still, there is not sufficient evidence to recommend consumption of resveratrol beyond the amount that can be obtained through dietary sources, and more human clinical tri- 
als are needed [35].

In spite of this, RV is still considered as a very promising "model compound". Perhaps more effective derivatives can be produced through effective chemical transformation, which shall be explored. It is worth mentioning that a recent study shows that polydatin, precursor of RV, can remove more hydroxyl radicals than RV in vitro. Therefore, the synthesis of RV analogues with higher bioavailability and solubility can increase the number of target biomolecules, better define the way of action and open up new prospects in the search and synthesis of new drug for the treatment of neurodegenerative diseases.

\section{References}

[1] Sparvoli, F., Martin, C., Scienza, A., Gavazzi, G. and Tonelli, C. (1994) Cloning and Molecular Analysis of Structural Genes Involved in Flavonoid and Stilbene Biosynthesis in Grape (Vitis vinifera L.). Plant Molecular Biology, 24, 743755. http://dx.doi.org/10.1007/BF00029856

[2] Wang, Q., Xu, J., Rottinghaus, G., Simonyi, A., Lubahn, D., Sun, G.Y. and Sun, A.Y. (2002) Resveratrol Protects against Global Cerebral Ischemic Injury in Gerbils. Brain Research, 958, 439-447. http://dx.doi.org/10.1016/S0006-8993(02)03543-6

[3] Rocha-Gonzalez, H., Ambriz-Tututi, M. and Granados-Soto, V. (2008) Resveratrol: A Natural Compound with Pharmacological Potential in Neurodegenerative Diseases. CNS Neuroscience \& Therapeutics, 14, 234-247. http://dx.doi.org/10.1111/j.1755-5949.2008.00045.x

[4] Selkoe, D.J. (2002) Alzheimer's disease Is a Synaptic Failure. Science, 298, 789-791. http://dx.doi.org/10.1126/science.1074069

[5] Tanzi, R.E. (2005) The Synaptic A $\beta$ Hypothesis of Alzheimer Disease. Nature Neuroscience, 8, 977-979. http://dx.doi.org/10.1038/nn0805-977

[6] Saraqoni, L., Hernandez, P. and Maccioni, R.B. (2000) Differential Association of Tau with Subsets of Microtubules Containing Posttranslationally-Modified Tubulin Variants in Neuroblastoma Cells. Neurochemical Research, 25, 5970. http://dx.doi.org/10.1023/A:1007587315630

[7] Smith, M.J., Crowther, R.A. and Goedert, M. (2000) The Natural Osmolytetrimethylamine N-Oxide (TMAO) Restores the Ability of Mutant Tau to Promote Microtubule Assembly. FEBS Letters, 484, 265-270. http://dx.doi.org/10.1016/S0014-5793(00)02169-4

[8] Takashima, A., Honda, T., Yasutake, K., et al. (1998) Activation of Tau Protein Kinasel/Glycogen Synthase Kinase-3Beta by Amyloid Beta Peptide (25-35) Enhances Phosphorylation of Tau in Hippocampal Neurons. Neuroscience Research, 31, 317-323. http://dx.doi.org/10.1016/S0168-0102(98)00061-3

[9] Cuello, A.C. (2005) Intracellular and Extracellular A $\beta$, a Tale of Two Neuropathologies. Brain Pathology, 15, 66-71. http://dx.doi.org/10.1111/j.1750-3639.2005.tb00101.x

[10] Ethell, D.W. and Buhler, L.A. (2003) Fas Ligand-Mediated Apoptosis in Degenerative Disorders of the Brain. Journal of Clinical Immunology, 23, 439-446. http://dx.doi.org/10.1023/B:JOCI.0000010420.96419.a8

[11] Dudek, H., Datta, S.R., Franke, T.F., Birnbaum, M.J., Yao, R., Cooper, G.M., Segal, R.A., Kaplan, D.R. and Greenberg, M.E. (1997) Regulation of Neuronal Survival by the Serine-Threonine Protein Kinase Akt. Science, 275, 661665. http://dx.doi.org/10.1126/science.275.5300.661

[12] Gertz, M., Nquyen, G.T., Fischer, F., et al. (2012) A Molecular Mechanism for Direct Sirtuin Activation by Res- veratrol. PLoS ONE, 7, e49761. http://dx.doi.org/10.1371/journal.pone.0049761

[13] Feng, Y., Wang, X.P., Yang, S.G., et al. (2009) Resveratrol Inhibits Beta-Amyloid Oligomeric Cytotoxicity But Does Not Prevent Oligomer Formation. Neuro Toxicology, 30, 986-995. http://dx.doi.org/10.1016/j.neuro.2009.08.013

[14] Cohen, T.J., Guo, J.L., Hurtado, D.E., Kwong, L.K., Mills, I.P., Trojanowski, J.Q. and Lee, V.M.Y. (2011) The Acetylation of Tau Inhibits Its Function and Promotes Pathological Tau Aggregation. Nature Communications, 2, Article No. 252. http://dx.doi.org/10.1038/ncomms1255

[15] Vonsattel, J.P. and Figlia, M.D. (1998) Huntington Disease. Journal of Neuropathology \& Experimental Neurology, 57, 369-384. http://dx.doi.org/10.1097/00005072-199805000-00001

[16] MacDonald, M.E., Ambrose, C.M., Duyao, M.P., et al. (1993) A Novel Gene Containing a Trinucleotide Repeat That Is Expanded and Unstable on Huntington's Disease Chromosomes. Cell, 72, 971-983. http://dx.doi.org/10.1016/0092-8674(93)90585-E

[17] Lajoie, P. and Snapp, E.L. (2010) Formation and Toxicity of Soluble Polyglutamine Oligomers in Living Cells. PLoS ONE, 5, e15245. http://dx.doi.org/10.1371/journal.pone.0015245

[18] Kumar, P., Padi, S.S., Naidu, P.S., et al. (2007) Cyclooxygenase Inhibition Attenuates 3-Nitropropionic Acid-Induced 
Neurotoxicity in Rats: Possible Antioxidant Mechanisms. Fundamental \& Clinical Pharmacology, 21, 297-306. http://dx.doi.org/10.1111/j.1472-8206.2007.00485.x

[19] Feng, X., Liang, N., Zhu, D., et al. (2013) Resveratrol Inhibits $\beta$-Amyloid-Induced Neuronal Apoptosis through Regulation of SIRT1-ROCK1 Signaling Pathway. PLOS ONE, 8, e59888. http://dx.doi.org/10.1371/journal.pone.0059888

[20] Bae, B.I., Xu, H., Igarashi, S., et al. (2005) P53 Mediates Cellular Dysfunction and Behavioral Abnormalities in Huntington's Disease. Neuron, 47, 29-41. http://dx.doi.org/10.1016/j.neuron.2005.06.005

[21] Luo, J., Nikolaev, A.Y., Imai, S., Chen, D., Su, F., Shiloh, A., Guarente, L. and Gu, W. (2001) Negative Control of P53 by Sir2Alpha Promotes Cell Survival under Stress. Cell, 107, 137-148. http://dx.doi.org/10.1016/S0092-8674(01)00524-4

[22] Zhou, S., Kachhap, S. and Singh, K.K. (2003) Mitochondrial Impairment in P53-Deficient Human Cancer Cells. Mutagenesis, 18, 287-292. http://dx.doi.org/10.1093/mutage/18.3.287

[23] Canto, C. and Auxerx, J. (2012) Targeting Sirtuin 1 to Improve Metabolism: All You Need Is NAD(+)? Pharmacological Reviews, 64, 166-187. http://dx.doi.org/10.1124/pr.110.003905

[24] Choi, K.M., Lee, H.L., Kwon, Y.Y., Kang, M.S., Lee, S.K. and Lee, C.K. (2013) Enhancement of Mitochondrial Function Correlates with the Extension of Lifespan by Caloric Restriction and Caloric restriction Mimetics in Yeast. Biochemical and Biophysical Research Communications, 441, 236-242. http://dx.doi.org/10.1016/j.bbrc.2013.10.049

[25] Barbato, D.L., Baldelli, S., Pagliei, B., et al. (2012) Caloric Restriction and the Nutrient-Sensing PGC-1 $\alpha$ in Mitochondrial Homeostasis: New Perspectives in Neurodegeneration. International Journal of Cell Biology, 2012, Article ID: 759583 .

[26] de Rijk, M.C., Tzourio, C., Breteler, M.M., Dartigues, J.F., Amaducci, L., Lopez-Pousa, S., Manubens-Bertran, J.M., Alpérovitch, A. and Rocca, W.A. (1997) Prevalence of Parkinsonism and Parkinson's Disease in Europe: The EUROPARKINSON Collaborative Study. European Community Concerted Action on the Epidemiology of Parkinson's Disease. Journal of Neurology, Neurosurgery, and Psychiatry, 62, 10-15. http://dx.doi.org/10.1136/jnnp.62.1.10

[27] Blesa, J., Phani, S., Jackson-Lewis, V., et al. (2012) Classic and New Animal Models of Parkinson's Disease. Journal of Biomedicine and Biotechnology, 2012, Article ID: 845618.

[28] Ferretta, A., Gaballo, A., Tanzarella, P., et al. (2014) Effect of Resveratrol on Mitochondrial Function: Implications in Parkin-Associated Familiar Parkinson's Disease. Biochimica et Biophysica Acta (BBA): Molecular Basis of Disease, 1842, 902-915. http://dx.doi.org/10.1016/j.bbadis.2014.02.010

[29] Vaarmann, A., Gandhi, S. and Abramov, A.Y. (2010) Dopamine Induces $\mathrm{Ca}^{2+}$ Signaling in Astrocytes through Reactive Oxygen Species Generated by Monoamine Oxidase. Journal of Biological Chemistry, 285, 25018-25023. http://dx.doi.org/10.1074/jbc.M110.111450

[30] Surmeier, D.J., Guzman, J.N., Sanchez-Padilla, J. and Goldberg, J.A. (2011) The Origins of Oxidant Stress in Parkinson's Disease and Therapeutic Strategies. Antioxidants \& Redox Signaling, 14, 1289-1301. http://dx.doi.org/10.1089/ars.2010.3521

[31] Duncan, R.S., Goad, D.L, Grillo, M.A., Kaja, S., Payme, A.J. and Koulen, P. (2010) Control of Intracellular Calcium Signaling as a Neuroprotective Strategy. Molecules, 15, 1168-1195. http://dx.doi.org/10.3390/molecules15031168

[32] Donmez, G., Arun, A., Chung, C.Y., McLean, P.J., Lindquist, S. and Guarente, L. (2012) SIRT1 Protects against Alpha-Synuclein Aggregation by Activating Molecular Chaperones. The Journal of Neuroscience, 32, 124-132. http://dx.doi.org/10.1523/JNEUROSCI.3442-11.2012

[33] Li, D.W., Liu, Z.Q., Chen, W., Yao, M. and Li, G.R. (2014) Association of Glycogen Synthase Kinase-3 $\beta$ with Parkinson's Disease (Review). Molecular Medicine Reports, 9, 2043-2050. http://dx.doi.org/10.3892/mmr.2014.2080

[34] Simao, F., Matte, A., Pagnussat, A.S., Netto, C.A. and Salbego, C.G. (2012) Resveratrol Prevents CA1 Neurons against Ischemic Injury by Parallel Modulation of Both GSK-3 $\beta$ and CREB through PI3-K/Akt Pathways. European Journal of Neuroscience, 36, 2899-2905. http://dx.doi.org/10.1111/j.1460-9568.2012.08229.x

[35] Vang, O., Ahmad, N., Baile, C.A., Baur, J.A., Brown, K., Csiszar, A., et al. (2011) What Is New for an Old Molecule? Systematic Review and Recommendations on the Use of Resveratrol. PLOS ONE, 6, e19881. http://dx.doi.org/10.1371/journal.pone.0019881 